\title{
A minimum curvature algorithm for tomographic reconstruction of atmospheric chemicals based on optical remote sensing
}

\author{
Sheng Li, Ke Du \\ Department of Mechanical and Manufacturing Engineering, University of Calgary, Calgary, AB T2N 1N4, Canada \\ 5 Correspondence to: Ke Du (kddu@ucalgary.ca)
}

\begin{abstract}
Optical remote sensing (ORS) combined with computerized tomography (CT) technique is a powerful tool to retrieve a two-dimensional concentration map over the area under investigation. But unlike the medical CT, the beam number used in ORS-CT is usually dozens comparing to up to hundreds of thousands in the former, which severely limits the spatial resolution and the quality of the reconstructed map. This situation makes the 'smoothness' a priori information especially necessary for ORS-CT. Algorithms which produce smooth reconstructions include smooth basis function minimization (SBFM), grid translation and multiple grid (GT-MG), and low third derivative (LTD), among which the LTD algorithm is a promising one with fast speed and simple realization. But its characteristics and the theory basis are not clear. Moreover, the computation efficiency and the reconstruction quality need to be improved for practical applications. This paper employs two theories, i.e., Tikhonov regularization and spatial interpolation, to produce a smooth reconstruction by ORS-CT. Within the

15 two theories' frameworks, new algorithms can be explored in order to improve the performance. For example, we propose a new minimum curvature (MC) algorithm based on the variational approach in the theory of the spatial interpolation, which reduces the number of linear equations by half comparing to that in the LTD algorithm using the biharmonic equation instead of the smoothness seminorm. We compared our MC algorithm with the non-negative least square (NNLS), GT-MG, and LTD algorithms using multiple test maps. The MC and the LTD algorithms have similar performance on the reconstruction quality.

20 But the MC algorithm needs only about $65 \%$ computation time of the LTD algorithm. It is much simpler in realization than the GT-MG algorithm by using high-resolution grids directly during the reconstruction process to generate a high-resolution map immediately after one reconstruction process is done. Comparing to the traditional NNLS algorithm, it shows better performance in three aspects: (1) the nearness of reconstructed maps is improved by more than $50 \%$; (2) the peak location accuracy is improved by 1-2 m; and (3) the exposure error is improved by more than 10 times. The testing results show the effectiveness of the new algorithm based on the spatial interpolation theory. Similarly, other algorithms may also be formulated to address problems such as the over-smooth issue in order to further improve the reconstruction equality. The studies will promote the practical application of the ORS-CT mapping of atmospheric chemicals.
\end{abstract}




\section{Introduction}

Measuring the concentration distribution of atmospheric chemicals over a large area is required in many environmental applications, including locating hot spots or emission sources of air pollutants (Wu et al., 1999), understanding air pollutant dispersion and airflow patterns, quantifying emission rate or ventilation efficiency (Samanta and Todd, 2000; Belotti et al., 2003; Arghand et al., 2015). Traditional methods use multiple point samplers placed at various locations in the region under investigation, which is intrusive, time-consuming, and limited in temporal and spatial resolution (Cehlin, 2019). The advanced method is based on the combination of optical remote sensing (ORS) and computerized tomography (CT) techniques (ORS$\mathrm{CT}$ ). ORS is a powerful technique for air contaminant measurement, which can detect a large area in situ and provide near real-time information (Du et al., 2011). The path-integrated concentration (PIC) is measured along each path using techniques like open-path tunable diode laser (OP-TDL) or open-path Fourier transform infrared spectrometer (OP-FTIR). After multiple PICs are collected, the two-dimensional concentration map can be reconstructed by the CT technique (Hashmonay et al., 2001). Comparing to other techniques, ORS-CT is non-intrusive and fast. It also has good spatial resolution and can work continuously and automatically. These advantages make ORS-CT have the potential to be an excellent tool for investigating air dispersion problems under various conditions.

The reconstruction techniques have been well studied in medical CT. Theoretically, the true distribution can be determined exactly by Radon transformation approach, which requires infinite beams. In practice, the transformation approaches include back projection and filtered back projection (FBP) methods. Series-expansion-based methods are another type of approaches which estimate the true distribution by finding a finite set of numbers or superposition of a set of simple functions. In environmental applications, the number of beams in ORS-CT is usually less than 50 comparing to hundreds of thousands of beams used in the medical CT due to factors including cost, response, configuration, and other practical considerations. Therefore, only the series-expansion-based methods are applied in ORS-CT, which include two main types of approaches: pixel-based approach and basis-function-based approach. Pixel-based approach divides an area into pixels (grids) and assigns a concentration value to each pixel. The PIC is calculated by summarising the product of the pixel concentration and the length of the path in each pixel. A system of linear equations can be set up for multiple beams. The question is to find the best set of pixel concentrations. Basis-function approach assumes that the true distribution is determined by the superposition of a set of simple basis functions with unknown parameters. Possible basis functions include bilinear function, bivariate Gaussian function, etc (Giuli et al., 1999; Hashmonay et al., 1999). A system of non-linear equations is set up for multiple beams. The question is to find the best set of parameters. For both approaches, the inverse questions are solved by minimizing the error function constructed based on some criteria (Price et al., 2001), including minimizing the $\mathrm{L}_{2}$ norm of error (finding a leastsquares (LS) solution), maximum likelihood (ML), maximum entropy, etc. In pixel-based reconstruction, the inverse problem is linear but usually ill-posed. The number of equations is very large. Numerical iterative techniques are used to estimate the solution, including algebraic reconstruction techniques (ART), steepest descent, conjugate gradient (CG), and expectationmaximization (EM) (Tsui et al., 1991; Todd and Ramachandran, 1994; Drescher et al., 1996). In the basis-function-based 
approach, the inverse problem is usually non-linear. Stochastic optimization algorithms need to be used to find the global minimum (e.g., simulated annealing (SA)).

For ORS-CT mapping of atmospheric chemicals, literatures show that the smooth basis function minimization (SBFM) algorithm performs better than other algorithms (Drescher et al., 1996). This is because the patterns of air dispersion are physically smooth in shape. Therefore, an algorithm converging toward smooth concentration distribution consistent with the path-integrated data is a rational choice (Cehlin, 2019). However, SBFM is highly computation-intensive due to the non-linear optimization, which was reported to be about 100 times slower than pixel-based algorithms (Price et al., 2001). This makes it unfavorable for rapid reconstruction, which is usually required in industrial applications such as monitoring chemical plants. A representative pixel-based algorithm is the non-negative least square (NNLS), which is also the algorithm used in the USEPA

70 OTM-10 for horizontal radial plume mapping (HRPM) measurements (EPA, 2005). It achieves similar results as the multiplicative algebraic reconstruction technique (MART), but with shorter computing time (Hashmonay et al., 1999). The traditional pixel-based algorithms, although suitable for rapid CT, have poor performance comparing to SBFM algorithm (Wu and Chang, 2011). This is mainly due to the coarse grid resolution limited by the beam number. Because the algorithm requires the number of grids to be less than or equal to the number of beams (Hashmonay, 2012).

75 To improve the performance of traditional pixel-based algorithms, there are two reported approaches used in ORS-CT. One method is the 'low third derivative' (LTD) algorithm, which adds the third derivative at each pixel as a smoothness restriction (Price et al., 2001). The LTD algorithm was reported to work nearly as well as SBFM algorithm, but was about 100 times faster (Price et al., 2001). Another method is the 'grid translation' (GT) algorithm, which shifts the basis grid by different distances (e.g., 1/3, 2/3 width of the basis grid) horizontally and vertically while keeping the basis grid fixed (Verkruysse and

80 Todd, 2004). The performance is improved by averaging the reconstruction results after each shifting. There is also an improved version called 'grid-translation and multi-grid' (GT-MG), which applies the GT algorithm at different basis grid resolutions (Verkruysse and Todd, 2005). This method has been successfully used with maximum likelihood expectation maximization (MLEM) to improve the reconstruction accuracy, especially in determining the peak location and value (Cehlin, 2019).

85 The successes of these algorithms show the necessary of applying the smoothness restriction to the ORS-CT gas mapping. Using the LTD algorithm, we can easily achieve a smooth reconstruction. However, like the Gaussian model used in the SBFM algorithm, the smoothness in the LTD algorithm is also achieved based on ideal model (quadratic functions). It is necessary to understand the characteristics of this model in order to apply the algorithm to specific application. But the theory basis of the LTD algorithm was not clearly given. When we tried to introduce the smoothness as a priori information to the reconstruction

90 process, we found that a smooth reconstruction could be achieved based on two well-established theories. The first one is the regularization theory originated from Tikhonov for solving the ill-posed inverse problem (Tikhonov and Arsenin, 1977; Rudin et al., 1992). The most well-known approach is the Tikhonov $\mathrm{L}_{2}$ regularization, which uses the $i t h$-order derivative of the function as a regularization factor and produces a smoothing effect on the resulting solution (Gholami and Hosseini, 2013). 
From the view of regularization theory, the LTD algorithm is a regularized algorithm which uses the third-order derivative. In fact, the first, second, and higher order derivatives can also produce smooth results. The second one is the interpolation theory based on which almost all algorithms introduce the smooth effect to the output. Specifically, the variational interpolation approach can produce a regularized inverse problem similar to Tikhonov regularization by using a smoothness seminorm. The seminorm consists of different order derivatives or their combinations. The solution to this inverse problem is a set of spline functions. For example, a bivariate smoothness seminorm with squares of second derivative leads to a thin plate spline (TPS) function. Based on the variational interpolation theory, the LTD algorithm is a special case of the regularized spline with tension (RST) (Mitasova et al., 1995).

In the framework of the regularized inversion and spatial interpolation theories, the characteristics of the derivative-based algorithms can be well explored. In this paper, we propose a new minimum curvature (MC) algorithm based on the variational interpolation. It improves the computation efficiency comparing to the LTD algorithm by using the corresponding biharmonic equation instead of the smoothness seminorm to construct the additional linear equation at each pixel of the map, the new MC algorithm reduces the number of equations to half of the LTD algorithm and eventually reduces the computation time. Another innovation of the new algorithm is to use much larger number of grids than the number of beams instead of the comparable numbers of grids and beams in the traditional methods. Comparing to the multiple grids and grid translation operations in GTMG algorithm, this approach offers the same performance but is much easier to realize. We compared the new MC algorithm with the NNLS, LTD, and GT-MG algorithms using multiple test maps. These tests showed the effectiveness of the new MC algorithm. This study also demonstrates the feasibility of introducing techniques from the Tikhonov regularization and spatial interpolation to the ORS-CT method for mapping atmospheric chemicals.

\section{Materials and methodologies}

\subsection{ORS-CT and beam geometry}

115 The area of the test field is $40 \mathrm{~m} \times 40 \mathrm{~m}$. We use the open-path tunable diode laser (OP-TDL) as the ORS analyzer, which is installed on a scanner and aims at multiple retroreflectors by scanning periodically and continuously. The beam geometry can be categorized into overlapped and non-overlapped beam geometry based on the way the beams are deployed. To compare with the results of GT-MG algorithm, we use an overlapping beam configuration similar to the one used in Verkruysse and Todd (2005).

120 As shown in Fig. 1, four TDL analyzers are located at the four corners of the test field. The retroreflectors are evenly distributed on the edges of the field. The total beam number is 38. For traditional pixel-based algorithm, the number of grids should be less than or equal to the beam number. Therefore, we divide the test field into $6 \times 6$ grids. The concentration within each grid is assumed to be uniform. 


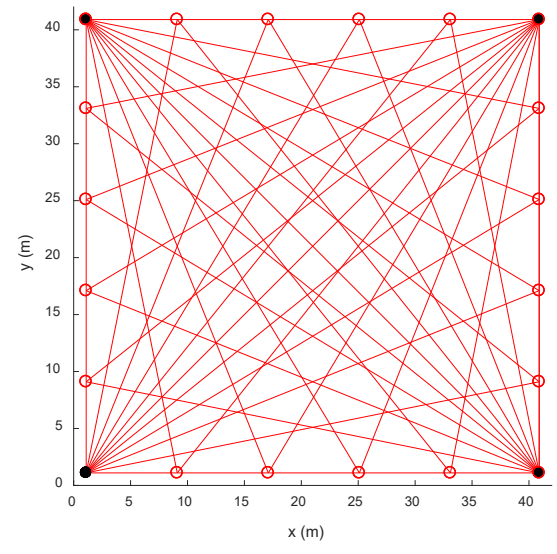

(a)

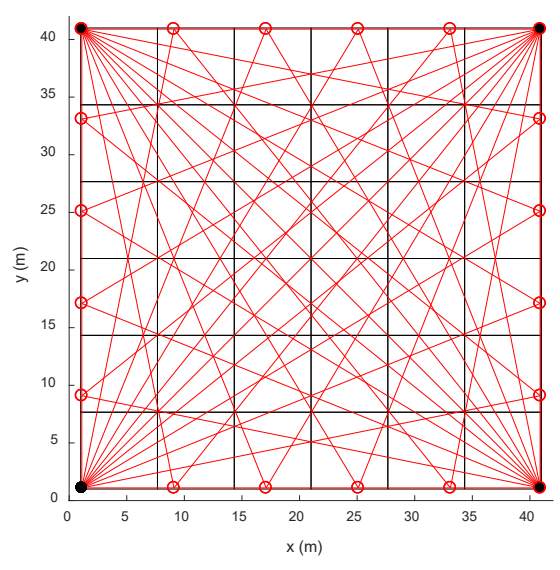

(b)

Figure 1. The test field and beam configuration. (a) beam geometry; (b) beam geometry with $6 \times 6$ grids.

At each retroreflector, the path-integrated concentration (PIC) is measured. The PIC of the $i$-th beam and the system of linear equation for all the paths are

$p_{i}=\sum_{j=1}^{n} L_{i j} c_{j}, \quad c_{j} \geq 0$

$\boldsymbol{p}=\boldsymbol{L c}, \quad c_{j} \geq 0$

where $p$ is the PIC, $i, j$ is the index of path number and cell number respectively, $L_{i j}$ is the beam length of the $i t h$ beam in the $j$ th cell, $c_{j}$ is the concentration of the $j$ th cell. This constrained linear inverse problem is solved by iterative algorithms of NNLS (Lawson and Janson, 1995).

\subsection{Tikhonov regularization and LTD algorithm}

135 Tikhonov $\mathrm{L}_{2}$ regularization is the most well-known regularization technique. Its form can be written as the following minimization problem ${ }^{21}$

$\underset{\boldsymbol{c} \in \mathbb{R}^{n}}{\arg \min }\left\{\|\boldsymbol{L} \boldsymbol{c}-\boldsymbol{p}\|_{2}^{2}+\mu\left\|\boldsymbol{R}_{i} \boldsymbol{c}\right\|_{2}^{2}\right\}$

where $\mu$ is the regularization parameter controlling the conditioning of the problem, matrix $\boldsymbol{R}_{i}$ is the regularization operator, which is typically an approximation of the $i t h$-order derivative operator.

The LTD algorithm uses the third-order derivative operator. Assuming the grid indices in $\mathrm{x}, \mathrm{y}$ directions are $i, j$, the third-order derivative at y-direction is

$\frac{d^{3} c}{d j^{3}}=c_{i, j+2}-3 c_{i, j+1}+3 c_{i, j}-c_{i, j-1}$

The third-order derivative operator in matrix form is 
$145 \quad \boldsymbol{R}_{3}=\left[\begin{array}{ccccccc}-1 & 3 & -3 & 1 & & & \\ & -1 & 3 & -3 & 1 & & \\ & & & \ddots & & & \\ & & & & & & \\ & & 1 & 3 & -3 & 1 & \\ & & & 1 & 3 & -3 & 1\end{array}\right] \in \mathbb{R}^{(m-3) \times n}$

For grids at the edges, we use the second-order derivative and first-order derivative. The regularization parameter is set to be inversely propositional to the grid length. By setting the derivative to zero, the algorithm introduces two linear equations at each grid. The resulted system of linear equations is over-determined, which can be solved by NNLS algorithm.

\subsection{Variational approach and minimum curvature algorithm}

The variational approach for interpolation is to minimize the following problem

$\underset{\boldsymbol{c} \in \mathbb{R}^{n}}{\arg \min }\left\{\|\boldsymbol{L} \boldsymbol{c}-\boldsymbol{p}\|_{2}^{2}+\omega|\boldsymbol{c}|^{2}\right\}$

where $\omega$ is a positive weight and $|\boldsymbol{c}|^{2}$ denotes the square of smoothness seminorm. The seminorm can be defined in various forms. The commonly used ones are the first, second, third derivatives or their combinations. The solutions of the minimizing problems are spline functions.

Based on different seminorms, we can formulate many different reconstruction algorithms. As a demonstration, we propose the minimum curvature (MC) algorithm, which uses the seminorm equal to the total square curvature:

$|\boldsymbol{c}|^{2}=\iint\left(\frac{\partial^{2} c}{\partial x^{2}}+\frac{\partial^{2} c}{\partial y^{2}}\right)^{2} d x d y$

The seminorm can be calculated at each pixel using the finite difference approach. Each item in the summation is set to be

zero to generate an equation at that pixel. Multiple items will lead to multiple equations at one pixel. This is how the LTD algorithm does to add additional equations. In this paper, however, we will only add one equation at each pixel to reduce the number of equations. This is done by using the corresponding Euler-Lagrange differential equation to the minimizing problem (Briggs, 1974). According to the minimum curvature principle, the minimization can be carried out by solving the biharmonic equation.

$165 \frac{\partial^{4} c}{\partial x^{4}}+2 \frac{\partial^{4} c}{\partial x^{2} \partial y^{2}}+\frac{\partial^{4} c}{\partial y^{4}}=0$

The corresponding finite difference equation is

$$
\begin{gathered}
c_{i+2, j}+c_{i, j+2}+c_{i-2, j}+c_{i, j-2} \\
+2\left(c_{i+1, j+1}+c_{i-1, j+1}+c_{i+1, j-1}+c_{i-1, j-1}\right) \\
-8\left(c_{i+1, j}+c_{i-1, j}+c_{i, j-1}+c_{i, j+1}\right)+20 c_{i, j}=0
\end{gathered}
$$


Therefore, one equation is set up for each grid instead of two equations in the LTD algorithm. For grids on the edges, we set the first and second derivatives to be zeros. The weight is set to be inversely propositional to the grid length. The resulted constrained system of linear equations is over-determined and can be solved by NNLS algorithm.

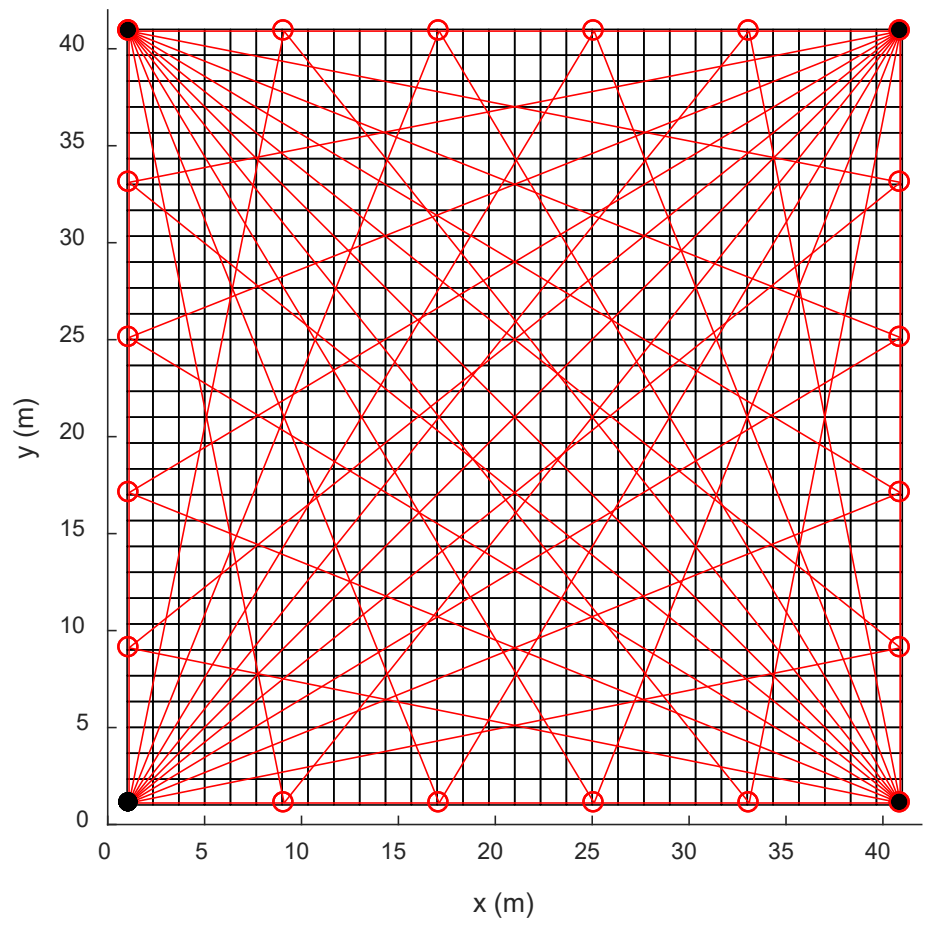

Figure 2. Beam geometry with $30 \times 30$ grids.

For pixel-based reconstruction algorithms, the number of grids (unknowns) should be no more than the number of beams (equations) in order to get a well-posted system of linear equations. Because there are only dozens beams in an ORS-CT

175 application, the grid resolution is very coarse. The GT algorithm is one way to increase the resolution. But it needs several steps to complete the whole translation. And the high-resolution map is generated after each reconstruction process. In the MC algorithm, we use very high-resolution grids directly before the reconstruction. Because there is one derivative restriction at each pixel, the resulted system of linear equations is determined. As shown in Fig. 2, $30 \times 30$ grids are used in the MC algorithm instead of the $6 \times 6$ grids in the traditional NNLS algorithm. The smoothness feature of the MC algorithm will guarantee the 180 smooth effect between all the pixels. This smooth effect is similar to the interpolation applied after the reconstruction process, except that the interpolation is achieved automatically when the inverse problem is solved. 


\subsection{Test concentration data}

The NNLS, LTD, and MC algorithms were compared using multiple test maps. The results were also compared with the GTMG algorithm. In order to do this, we set up test conditions similar to that used in Verkruysse and Todd (2005). The source distribution is defined by a bivariate Gaussian distribution

$c(x, y)=Q \exp \left[-\left(\frac{\left(x-x_{0}\right)^{2}}{\sigma_{x}^{2}}+\frac{\left(y-y_{0}\right)^{2}}{\sigma_{y}^{2}}\right)\right]$

where $Q(0$ to $40 \mathrm{ppm})$ is the source strength, $x_{0}, y_{0}(0$ to $40 \mathrm{~m})$ is the peak location, $\sigma$ is the width of the peak $(2.8,4.2,5.7$, or 7.1).

The source number varies from 1 to 5. For each source number, 100 maps were generated by randomly setting the source strength, location, and peak width.

\subsection{Evaluation of reconstruction quality}

A conventional image quality measure called nearness is used to describe the discrepancy between the original maps and the reconstructed maps. Nearness evaluates errors over all the grid cells in the map (Verkruysse and Todd, 2005)

Nearness $=\sqrt{\frac{\sum_{i}^{m \times n}\left(c_{i}^{*}-c_{i}\right)^{2}}{\sum_{i}^{m \times n}\left(c_{i}^{*}-c_{a v g}^{*}\right)^{2}}}$

195 where $m, n$ are the grid divisions on the $x, y$ direction of the map, $c_{i}^{*}$ is the synthetic value of concentration in the $i$ th grid on the map generated by the Gaussian distribution model, $c_{i}$ is the estimated value for the $i$ th grid in the map, $c_{a v g}^{*}$ is the mean concentration of all the grids in the map. A nearness value of zero implies a perfect match.

The effectiveness of locating the emission source is evaluated by peak location error, which calculates the distance between the true and reconstructed peak locations.

200 Peak location error $=\sqrt{\left(x_{r}-x_{0}\right)^{2}+\left(y_{r}-y_{0}\right)^{2}}$

where $x_{r}, y_{r}$ are the peak locations in the reconstruction map. For multiple peaks, only the location of the highest peak was calculated.

Exposure error percent is used to evaluate how well average concentrations in the whole field are reconstructed. It can reflect the accuracy of measuring chemical air emissions and emission rates from fugitive sources such as agricultural sources and landfills (Verkruysse and Todd, 2004)

Exposure error $\%=\left|\frac{\sum_{i}^{m \times n} c_{i}^{*}-\sum_{i}^{m \times n} c_{i}}{\sum_{i}^{m \times n} c_{i}^{*}}\right| \times 100 \%$

\section{Results and discussions}

In these tests, the traditional NNLS algorithm uses $6 \times 6$ grids, while the LTD and MC algorithms both use $30 \times 30$ grids. The results of the GT-MG algorithm are from Verkruysse and Todd (2005). They used a maximum basis grid resolution of $10 \times$ 
10 with $1 / 4$ grid size as translation distance. Note that the test conditions are not exactly the same as the ones used by the GTMG algorithm. Therefore, the results of the GT-MG algorithm are listed as a reference. Only the nearness results of the GTMG algorithm are compared because It did not give the peak location error, and the exposure error was calculated only in a small area near the peak instead of the whole map in this paper. The original resolution of the reconstruction map by the NNLS algorithm is too coarse $(6.7 \mathrm{~m})$. In order to determine the peak locations more accurately, all the reconstructed concentration maps by NNLS algorithm are interpolated by spline function using a resolution of $0.5 \mathrm{~m}$.

Table 1. Mean and standard deviation of nearness.

\begin{tabular}{lllll}
\hline Source number & NNLS & LTD & MC & GT-MG \\
\hline 1 & $0.40(0.21)$ & $0.13(0.08)$ & $0.11(0.07)$ & $0.09(0.05)$ \\
2 & $0.38(0.16)$ & $0.15(0.07)$ & $0.13(0.06)$ & $0.16(0.07)$ \\
3 & $0.40(0.14)$ & $0.18(0.08)$ & $0.17(0.08)$ & $0.19(0.06)$ \\
4 & $0.40(0.12)$ & $0.20(0.08)$ & $0.19(0.08)$ & $0.25(0.08)$ \\
5 & $0.43(0.13)$ & $0.22(0.09)$ & $0.21(0.08)$ & $0.27(0.09)$
\end{tabular}

*: The results of the GT-MG algorithm are from Verkruysse and Todd (2005), whose test conditions are not exactly the same as the conditions in this paper.

The nearness is the most important measure of accuracy of the reconstructed map, which represents the reconstruction of peak heights, shapes, and the production of artifacts. The smaller the number of sources, the better the reconstruction quality. In Table 1, LTD, MC, and GT-MG algorithms generally reduce the nearness values by more than $50 \%$ of the values obtained by the NNLS algorithm. In the condition of one source, they can reduce the nearness by about $70 \%$ with respect to NNLS. LTD, MC, GT-MG algorithms show increasing trends as the source number increases, which implies that the performance of the algorithm is affected by the complicity of the underlying distribution. The nearness results of NNLS for different numbers of sources are almost the same because they are the results after spline interpolation. In fact, the original un-interpolated results also show increasing trends. The interpolation improves the results of the NNLS algorithm more than those of LTD and MC algorithms, which already use high-resolution grids. The overall performances of LTD, MC, and GT-MG algorithms are very similar, while the new MC algorithm performances slightly better.

Table 2. Mean and standard deviation of peak location error.

\begin{tabular}{llll}
\hline Source number & NNLS $(\mathrm{m})$ & LTD $(\mathrm{m})$ & MC $(\mathrm{m})$ \\
\hline 1 & $1.78(0.93)$ & $0.41(0.45)$ & $0.40(0.56)$ \\
2 & $4.88(8.21)$ & $1.97(5.98)$ & $1.62(4.81)$ \\
3 & $5.17(8.35)$ & $2.58(6.77)$ & $2.34(6.17)$ \\
4 & $8.40(11.53)$ & $5.22(10.28)$ & $5.58(10.76)$ \\
5 & $8.95(11.32)$ & $5.51(10.15)$ & $5.77(10.41)$ \\
\hline
\end{tabular}


230 In Table 2, LTD and MC algorithms show better performance in peak location error than the NNLS algorithm. They generally improve the accuracy of peak location by 1 to $2 \mathrm{~m}$. The errors of all the algorithms increase as the source number increase. Because only the highest peak was calculated, a large error may happen when there are two or more peaks that are comparable to each other in magnitude (see Fig. 3). Therefore, this measure is more meaningful when the source number is small.

Table 3. Mean and standard deviation of exposure error.

\begin{tabular}{llll}
\hline Source number & NNLS (\%) & LTD (\%) & MC (\%) \\
\hline 1 & $29.26(19.29)$ & $1.51(2.11)$ & $1.61(2.15)$ \\
2 & $19.91(11.99)$ & $1.05(1.26)$ & $1.09(1.19)$ \\
3 & $16.05(9.78)$ & $1.16(0.97)$ & $1.31(0.92)$ \\
4 & $12.99(6.92)$ & $1.15(1.04)$ & $1.24(1.00)$ \\
5 & $12.13(8.62)$ & $1.30(1.14)$ & $1.45(1.16)$ \\
\hline
\end{tabular}

235 In Table 3, we can see a significant improvement of exposure error by MC and LTD algorithms comparing to the NNLS algorithm. The exposure error reflects the accuracy of the overall emissions measurement other than the concentration distribution. The performances of LTD and MC algorithms are very similar to each other. Unlike the trends that the NNLS shows in the nearness and peak location error, its performance in exposure error becomes better with the increase of source number. A plausible cause of this phenomenon could be that the distribution becomes more uniform with larger number of sources, implying that the NNLS algorithm is more suitable for relatively uniform distributions.

Table 4. Mean and standard derivation of computation time.

\begin{tabular}{llll}
\hline Source number & LTD $(\mathrm{s})$ & MC $(\mathrm{s})$ & Ratio (MC/LTD) \\
\hline 1 & $11.08(14.27)$ & $8.06(10.03)$ & 0.73 \\
2 & $21.02(17.89)$ & $14.17(11.46)$ & 0.67 \\
3 & $34.44(19.93)$ & $22.76(13.61)$ & 0.66 \\
4 & $43.58(20.45)$ & $29.13(12.92)$ & 0.67 \\
5 & $59.48(21.98)$ & $38.74(15.05)$ & 0.65
\end{tabular}

In Table 4, the computation time for LTD and MC algorithms are compared. The computation time generally increase with the increase of source number. MC algorithm is faster than LTD algorithm because it has about half the number of the linear equations than that in LTD algorithm. The ratio result shows that the MC algorithm's computation time is about $65 \%$ of that 245 of the LTD algorithm when the source number is five. The trend of the ratio implies that the advantage of the MC algorithm becomes more obvious with the increasing complexity of the underlying distribution. 
https://doi.org/10.5194/amt-2021-122

Preprint. Discussion started: 7 May 2021

(c) Author(s) 2021. CC BY 4.0 License.

\section{Atmospheric Measurement Techniques \\ Discussions}
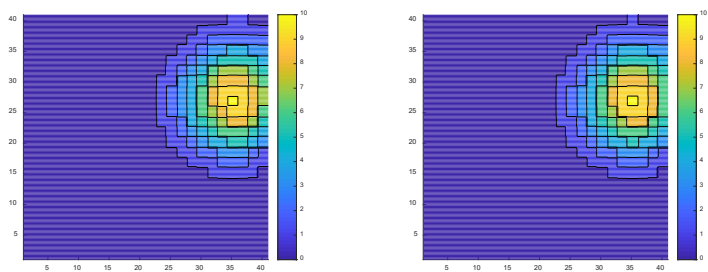

250 (b)
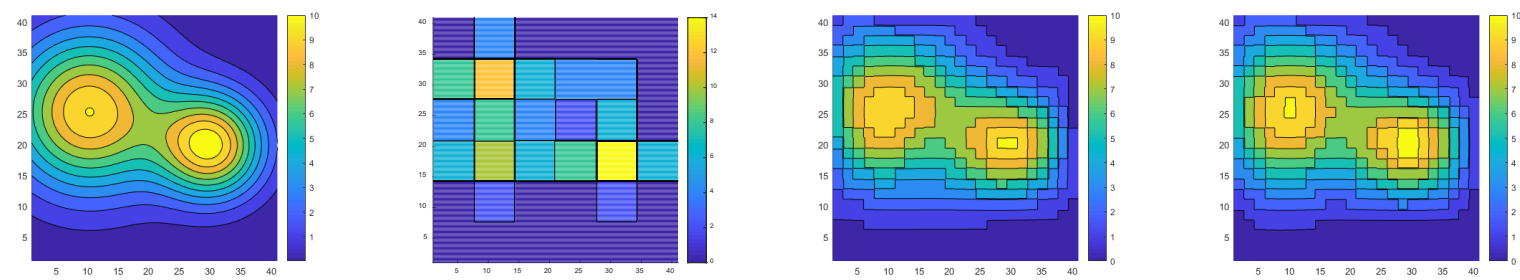

(c)
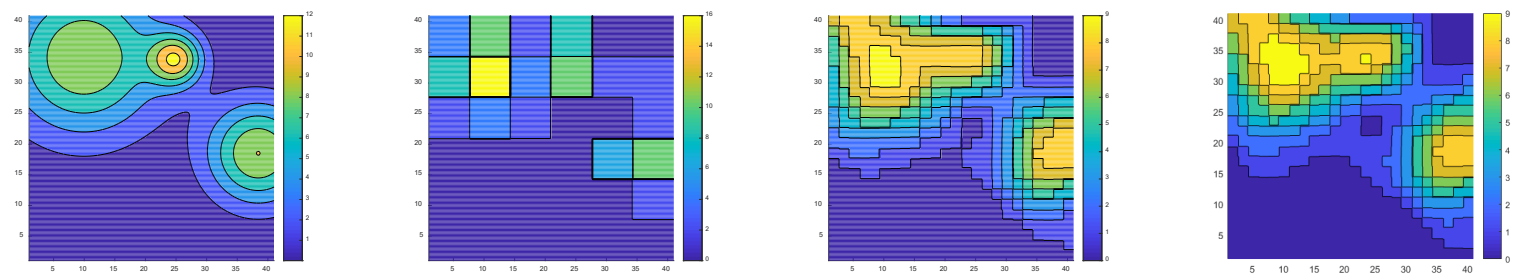

(d)
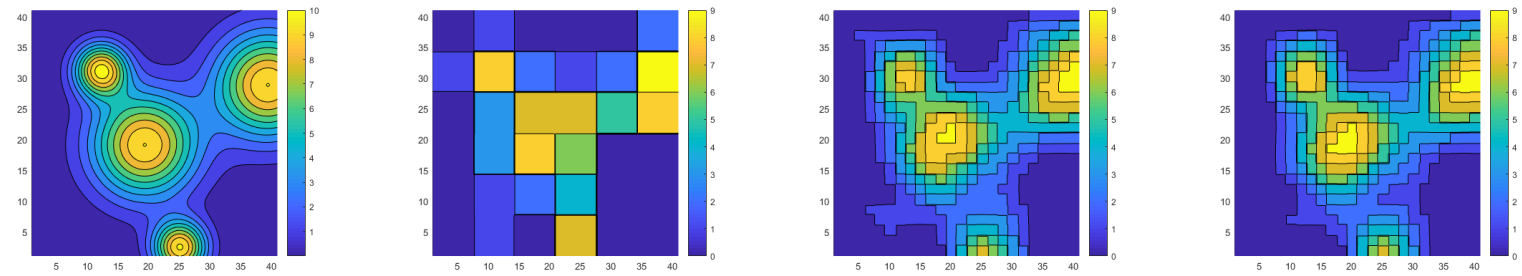

(e)
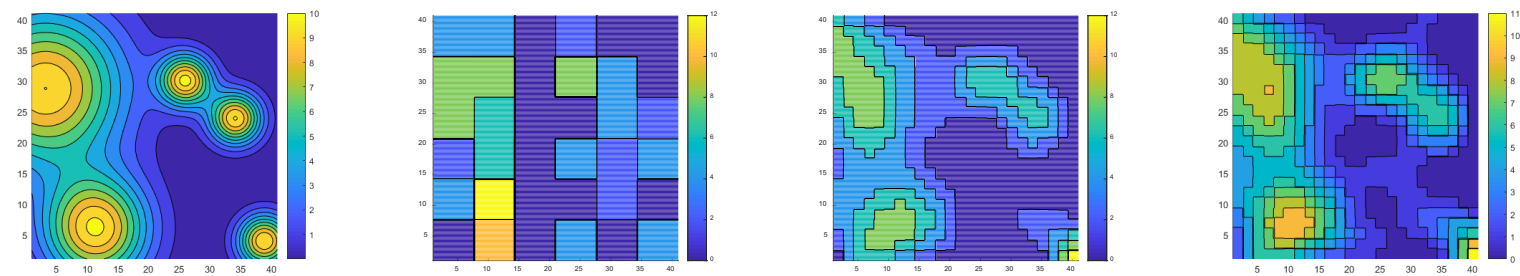

Figure 3. Original test maps (first column) and corresponding reconstructed maps using the NNLS (second column), LTD (third column), and MC (forth column) algorithms. (a) one source; (b) two sources; (c) three sources; (d) four sources; (e) five sources. 
Fig. 3 depicts some examples of the test maps and reconstructed maps generated by different algorithms under different source numbers. As indicated by the nearness results, LTD and MC algorithms show better accuracy than the NNLS algorithm in the shapes and source strengths. Because the differences in accuracy (i.e., nearness, peak distance) between LTD and MC algorithms are very small, more specific evaluations may be needed to compare them using more complicated and realistic conditions.

\section{Conclusion}

With the purpose of achieving a smooth reconstruction, we found that the Tikhonov regularization and the spatial interpolation theories can be applied to the ORS-CT inverse problems. Based on the variational approach in the interpolation theory, we proposed a new MC algorithm by using the corresponding biharmonic equation instead of the smoothness seminorm to construct an additional linear equation at each pixel of the map. The new algorithm improves the computation efficiency comparing to the LTD algorithm by reducing the number of linear equations by half. Comparing to the GT-MG algorithm, which achieves high-resolution reconstruction by shifting the low-resolution base grids and averaging the resulted maps, the new MC algorithm uses high-resolution grids directly during the reconstruction process to generate a high-resolution map immediately after the reconstruction is done. Therefore, it is much simpler in realization.

The MC, LTD, and NNLS algorithms were compared using multiple test maps with the GT-MG algorithm as a reference. The new MC algorithm shows almost the same performance as the LTD algorithm, but only needs about $65 \%$ of its computation time. The smoothness-related algorithms of LTD, MC, and GT-MG all show better performance than the traditional NNLS algorithm: the nearness of reconstructed maps is improved by more than $50 \%$, the peak distance accuracy is improved by $1-2$ $\mathrm{m}$, and the exposure error is improved by more than 10 times. Because the differences in accuracy (i.e., nearness, peak distance) between LTD and MC algorithms are very small, more specific evaluations may be needed to compare them using more complicated and realistic conditions.

These comparisons prove the feasibility of introducing theories of Tikhonov regularization and spatial interpolation techniques to mapping distribution of atmospheric chemicals using the ORS-CT techniques. With these theories, it is easier to understand the advantages and the drawbacks of the current algorithm. Common problems such as the over-smooth issue may be solved by testing more techniques in these theories and using them in the ORS-CT applications. We need to note that although the 285 smoothness is very good a priori information for the reconstruction problem, beam configuration and underlying concentration distribution are also important factors affecting the reconstruction equality. To further improve the reconstruction quality, extra a priori information based on the specific application may be added to the inverse problem. For example, the statistic information of the underlying distribution, or the information resulting from the fluid mechanics. 
Code and data availability. Data and code are available on request by contacting the authors.

Author contributions. KD was responsible for acquiring funding for this research. SL designed the algorithm and conducted the tests. SL and KD were both involved in data analysis. Both authors contributed to writing and editing the manuscript.

Competing interests. The authors declare that they have no conflict of interest.

Acknowledgements. The authors are grateful to the supports by the following grants: Discovery Grant from Natural Sciences and Engineering Research Council (NSERC) of Canada (RGPIN-2020-05223), John R. Evans Leaders Fund (JELF) and Infrastructure Operating Fund (IOF) from Canada Foundation for Innovation (CFI) (35468), University Research Grant Committee (URGC) seed grant from University of Calgary (1050666).

\section{References}

Arghand, T., Karimipanah, T., Awbi, H.B., Cehlin, M., Larsson, U., Linden, E.: An experimental investigation of the flow and comfort parameters for under-floor, confluent jets and mixing ventilation systems in an open-plan office. Building and Environment, 92, 48-60. DOI: 10.1016/j.buildenv.2015.04.019, 2015.

Belotti, C., Cuccoli, F., Facheris, L., Vaselli, O.: An application of tomographic reconstruction of atmospheric CO2 over a volcanic site based on open-path IR laser measurements. IEEE Transactions on Geoscience and Remote Sensing, 41:11, 2629-2637. DOI: 10.1109/TGRS.2003.815400, 2003.

Briggs, I.C., 1974. Machine contouring using minimum curvature. Geophysics, 39:1, 39. DOI: 10.1190/1.1440410.

Cehlin, M.: Mapping tracer gas concentrations using a modified Low Third Derivative method: numerical study. International Journal of Ventilation, 18:2, 136-151. DOI: 10.1080/14733315.2018.1462935, 2019.

Drescher, A.C., Gadgil, A.J., Price, P.N., Nazaroff, W.W.: Novel approach for tomographic reconstruction of gas concentration distributions in air: Use of smooth basis functions and simulated annealing. Atmospheric Environment, 30:6, 929-940. DOI: 10.1016/1352-2310(95)00295-2, 1996.

Du, K., Rood, M. J., Welton, E. J., Varma, R. M., Hashmonay, R. A., Kim, B. J., Kemme, M. R.: Optical Remote Sensing to Quantify Fugitive Particulate Mass Emissions from Stationary Short-Term and Mobile Continuous Sources: Part I. Method and Examples. Environ. Sci. \& Technol., 45, 658-665. DOI: 10.1021/es101904q, 2011.

EPA: Measurement of Fugitive Emissions at a Landfill Practicing Leachate Recirculation and Air Injection. EPA-600/R05/088, 2005.

Gholami, A., Hosseini, M.: A balanced combination of Tikhonov and total variation regularizations for reconstruction of piecewise-smooth signals. Signal Processing, 93:7, 1945-1960. DOI: 10.1016/j.sigpro.2012.12.008, 2013. 
Giuli, D., Facheris, L., Tanelli, S.: Microwave tomographic inversion technique based on stochastic approach for rainfall fields monitoring. IEEE Transactions on Geoscience and Remote Sensing, 37, 5, 2536-2555. DOI: 10.1109/36.789649, 1999.

Hashmonay, R.A., Natschke, D.F., Wagoner, K., Harris, D.B., Thompson, E.L, Yost, M.G.: Field Evaluation of a Method for Estimating Gaseous Fluxes from Area Sources Using Open-Path Fourier Transform Infrared. Environ. Sci. Technol., 35:11, 2309-2313. DOI: 10.1021/es0017108, 2001.

Hashmonay, R.A., Yost, M.G., Wu, C.F.: Computed tomography of air pollutants using radial scanning path-integrated optical remote sensing. Atmospheric Environment, 33, 267-274. DOI: 10.1016/S1352-2310(98)00158-7, 1999.

Hashmonay, R.A.: Theoretical evaluation of a method for locating gaseous emission hot spots. Journal of the Air and Waste Management Association, 58:8, 1100-1106. DOI: 10.3155/1047-3289.58.8.1100, 2012.

330 Lawson, C.L., Janson, R.J.: Solving least squares problems. Society for Industrial and Applied Mathematics: Philadelphia, 23, 158-165. DOI: 10.1137/1.9781611971217, 1995.

Mitasova, H., Mitas, L., Brown, W.M., Gerdes, D.P., Kosinovsky, I., Baker, T.: Modelling spatially and temporally distributed phenomena: new methods and tools for GRASS GIS, International Journal of Geographical Information Systems, 9:4, 433446. DOI: 10.1080/02693799508902048, 1995.

335 Price, P.N., Fischer, M.L., Gadgil, A.J., Sextro, R.G.: An algorithm for real-time tomography of gas concentrations, using prior information about spatial derivatives. Atmos. Environ., 35, 2827. DOI: 10.1016/S1352-2310(01)00082-6, 2001.

Rudin, L., Osher, S.J., Fatemi, E.: Non-linear total variation based noise removal algorithms. Physica D, 60, 259-268.

Samanta, A., Todd, L.A., 2000. Mapping chemicals in air using an environmental CAT scanning system: evaluation of algorithms. Atmospheric Environment, 34, 699-709. DOI: 10.1016/S1352-2310(99)00331-3, 1992.

340 Tikhonov, A.N., Arsenin, V.Y.: Solutions of Ill-posed Problems, Winston and Sons, Washington, 1977.

Todd L., Ramachandran, G.: Evaluation of Optical Source-Detector Configurations for Tomographic Reconstruction of Chemical Concentrations in Indoor Air, American Industrial Hygiene Association Journal, 55:12, 1133-1143, DOI: 10.1080/15428119491018204, 1994.

Tsui, B.M.W., Zhao, X., Frey, E.C., Gullberg, G.T.: Comparison between ML-EM and WLS-CG algorithms for SPECT image reconstruction. IEEE Transactions on Nuclear Science, 38:6, 1766-1772. DOI: 10.1109/23.124174, 1991.

Verkruysse, W., Todd L.A.: Improved method "grid translation" for mapping environmental pollutants using a twodimensional CAT scanning system. Atmospheric Environment, 38, 1801-1809. DOI: 10.1016/j.sigpro.2012.12.008, 2004.

Verkruysse, W., Todd, L.A.: Novel algorithm for tomographic reconstruction of atmospheric chemicals with sparse sampling. Environ Sci Technol., 39:7, 2247-2254. DOI: 10.1021/es035231v, 2005.

350 Wu, C.F., Chang, S.Y.: Comparisons of radial plume mapping algorithms for locating gaseous emission sources. Atmospheric Environment, 45, 1476-1482. DOI: 10.1016/j.atmosenv.2010.12.016, 2011. 
https://doi.org/10.5194/amt-2021-122

Preprint. Discussion started: 7 May 2021

(c) Author(s) 2021. CC BY 4.0 License.

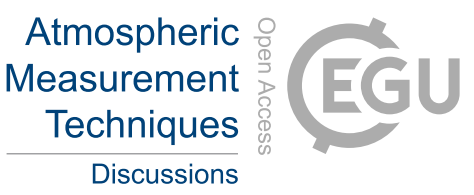

Wu, C.F., Yost, M.G., Hashmonay, R.A., Park, D.Y.: Experimental evaluation of a radial beam geometry for mapping air pollutants using optical remote sensing and computed tomography. Atmospheric Environment, 33, 4709-4716. DOI: 10.1016/S1352-2310(99)00218-6, 1999. 